\title{
Floral Meristem Development in Cranberry Apical Buds during Winter Rest and Its Implication on Yield Prediction
}

\author{
Jenny L. Bolivar-Medina ${ }^{1}$, Camilo Villouta ${ }^{2}$, Beth Ann Workmaster ${ }^{3}$, and Amaya Atucha ${ }^{4,5}$ \\ Department of Horticulture, University of Wisconsin-Madison, 1575 Linden Drive, Madison, WI \\ 53706
}

\begin{abstract}
AdDITIONAL INDEX wORDS. crop forecast dormancy, floral primordia, upright, Vaccinium macrocarpon
Abstract. The formation and development of floral meristems is key to fruit production. However, limited information regarding the development of floral buds during the dormant period of cranberry (Vaccinium macrocarpon) constrains the ability to forecast yield early and accurately. The objectives of this study were to characterize the development of floral meristems from fall to spring and to evaluate the number of floral meristems formed across different bud sizes and upright types, as well as their contribution to the fruit production of the next year. Apical buds of different sizes on vegetative and fruiting uprights were tagged and collected periodically from fall to spring for histological study. An extra set of tagged buds was left in the field to evaluate their flower and fruit production. Five stages of floral development were identified based on the concentric differentiation of organ primordia. Large buds from vegetative uprights developed earlier, had a higher number of floral meristems, and became fruiting uprights; they had the highest number of flowers and fruit. Buds from fruiting uprights had the lowest number of floral meristems and delayed development; subsequently, they had the lowest number of fruit per upright. Our results provide evidence of active floral meristem differentiation during fall and winter, as well as differences in the timing and development stage according to bud size. In addition, our study shows that upright types and bud sizes influence the fruit production of the following year; therefore, they should be considered in cranberry crop forecasting models.
\end{abstract}

Crop forecasting is a highly desirable tool for fruit production. For cranberry, early and accurate yield prediction would benefit handlers and processors to plan for crop volumes and fruit prices; most importantly, it would allow growers to customize inputs based on potential yield returns. The current yield prediction model for cranberry consists of evaluating the number of large apical buds per unit area in early fall; however, this model is highly inaccurate. Recently, DeVetter et al. (2015), in an effort to improve yield prediction, evaluated a model that included environmental and physiological factors, but they could only determine that berry number and size were the best predictors of yield. These factors are not useful for early crop forecasting, and large amounts of unexplained variations are associated with factors explaining the berry number (DeVetter et al., 2015).

Flower bud number and development are the most critical factors for accurate yield prediction (Aggelopoulou et al., 2011). Flower formation is a multistep process, from floral meristems differentiation to anthesis, and all of the steps are influenced by endogenous factors, such as resource allocation (Alvarez-Buylla et al., 2010; Liu et al., 2009; Polito et al.,

Received for publication 18 Mar. 2019. Accepted for publication 4 June 2019. We thank Dr. Sara E. Patterson for technical assistance with the histology work; Maria Kamenetsky from the University of Wisconsin-Madison, College of Agricultural and Life Sciences (UW CALS) Statistical Consulting Group for assistance with the data analysis; Cranberry Creek Cranberries Inc. for allowing us to collect data and plant material from their farm; and the Wisconsin Cranberry Board and Cranberry Institute for their financial support.

${ }^{1}$ Research Associate.

${ }^{2}$ Graduate Student.

${ }^{3}$ Researcher.

${ }^{4}$ Assistant Professor.

${ }^{5}$ Corresponding author. E-mail: atucha@wisc.edu.
2002). Previous studies examining cranberry bud floral differentiation focused on the timing of the first appearance of floral primordia in summer (Bolivar-Medina et al., 2019; DeVetter et al., 2013a; Goff, 1901; Lacroix, 1926; Roberts and Struckmeyer, 1943). Lacroix (1926) described the floral meristem development process of older cranberry cultivars; however, a more complete study has not been performed for current high-yielding cultivars. Furthermore, the extent of cranberry floral meristem development during fall and winter and its effect on fruit production during the following season have not been determined.

Cranberry is a perennial woody vine native to North America that produces vertical stems, known as uprights, which grow from long runners. Newly formed uprights arise from vegetative axillary buds of runners, whereas older uprights grow each season from mixed (vegetative and floral meristems) or vegetative apical buds of extant uprights. Fruiting uprights produce leaves, flowers, and, subsequently, fruit, whereas vegetative uprights do not bear any fruit. Both upright types have the potential to set a mixed apical bud in new cultivars (Roper and Vorsa, 1997; Trehane, 2004; Vorsa and JohnsonCicalese, 2012). A wide range of terminal bud size has been observed, particularly in newer high yielding cultivars. However, the implications of bud size and the type of upright (i.e., vegetative or fruiting) have never been considered during cranberry crop forecasting, and they could potentially account for the large amount of unexplained variations in crop yield forecasting models.

The objectives of the present study were as follows: to describe the development of floral meristems in cranberry mixed terminal buds; to evaluate the extent of floral meristem development of small, medium, and large mixed buds of vegetative and fruiting uprights from fall to spring; and to 
evaluate the contributions of mixed bud sizes and upright types to fruit production.

\section{Materials and Methods}

Plant material. Cranberry uprights were collected from a bed at a commercial cranberry farm located near Necedah, WI

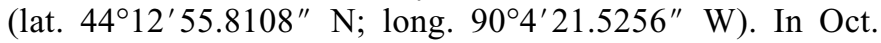
2015, immediately before fruit harvesting (preharvest), uprights of the cultivar HyRed were tagged in the field based on upright type and bud size. Fruiting uprights (F) and vegetative uprights (V) with large, medium, and small apical buds (Fig. 1) were tagged. Fruiting uprights refer to those uprights that produce flowers and develop fruit. Once fruit were harvested, fruiting uprights were identified by the presence of persistent pedicels. Vegetative uprights produce only leaves during the growing season. Apical bud width was characterized as large $(\mathrm{L} ;>1 \mathrm{~mm})$, medium $(\mathrm{M} ; 0.6-1 \mathrm{~mm})$, and small $(\mathrm{S} ; \leq 0.5 \mathrm{~mm})$. Upright type and bud size descriptors were combined as the following designations: VL, VM, VS, FM, and FS; the category FL was not used because this upright-bud size combination was rarely found (Fig. 1). A total of 360 uprights were tagged.

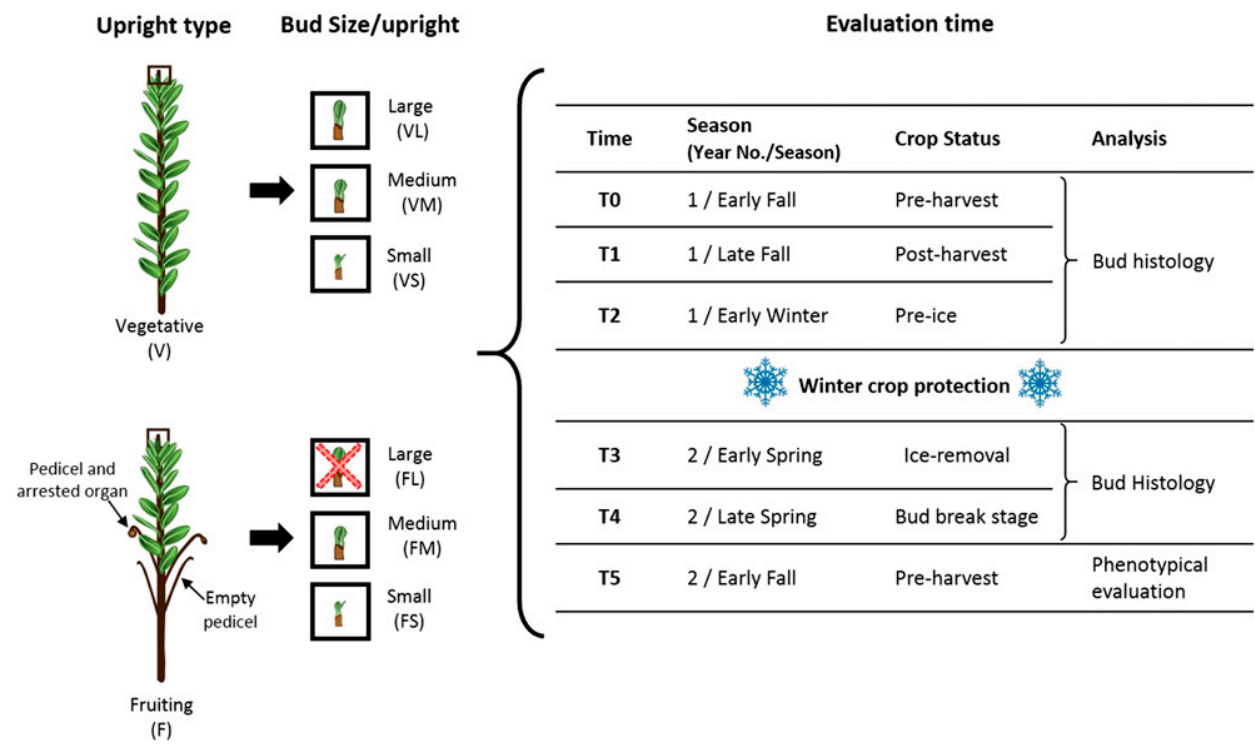

Fig. 1. Representation of cranberry plant material used and evaluations performed in 2015 (year 1) and 2016 (year 2), as well as sample collection and evaluation times, crop status, and type of analysis performed. Vegetative (V) and fruiting (F) uprights were tagged in the field according to the corresponding bud width sizes studied: large $(\mathrm{L} ;>1.0 \mathrm{~mm}) ;$ medium $(\mathrm{M} ; 0.6-1.0 \mathrm{~mm})$; and small $(\mathrm{S} ; \leq 0.5 \mathrm{~mm})$. No large buds were found on fruiting uprights (FL), represented by the " $\mathrm{X}$ " symbol. Histological analysis was performed for buds collected from T0 (year 1, early fall) to T4 (year 2, late spring). Phenotypical evaluations were performed at T5 (year 2, early fall) for current uprights grown from buds tagged in the field at $\mathrm{T} 0$.

Table 1. Analysis of variance (type III) of the number of floral meristems per bud of cranberry during the dormant period from T0 (year 1, early fall) to T4 (year 2, Spring 2016).

\begin{tabular}{lcrrc}
\hline Source & Sum of squares & df & $F$ & $P$ \\
\hline Intercept & 105.800 & 1 & 51.8568 & $1.180 \mathrm{e}-10^{* * *}$ \\
Bud category & 174.640 & 4 & 21.3995 & $9.662 \mathrm{e}-13^{* * *}$ \\
Collection time & 5.300 & 4 & 0.6494 & 0.6286031 \\
Bud category: Collection time & 90.968 & 16 & 2.7867 & $0.0009857^{* * *}$ \\
Residuals & 201.983 & 99 & & \\
\hline
\end{tabular}

${ }^{* * *}$ Significant at $P \leq 0.001$.
A set of 10 tagged uprights per upright type and bud size category (VL, VM, VS, FM, and FS) was collected five times (T0-T4) during one dormant season from Fall 2015 (year 1) to Spring 2016 (year 2) for bud histological analysis (Fig. 1). The first set of uprights was collected on 11 Oct. [T0 (year 1, early fall)], when fruit were mature and before harvest. The second collection was obtained postharvest on 9 Nov. [T1 (year 1, late ], when the foliage had developed its characteristic red 1 , early winter)], when the daily maximum and minimum temperatures were below $0{ }^{\circ} \mathrm{C}$ but before the The fourth collection was obtained on 9 Mar. melted from the collection occurred on 4 May [T4 (year 2, late spring)], at the time of bud swell. A sixth set of tagged uprights was kept in each collection date, harvested uprights were immediately placed on ice for transport and then stored at $4{ }^{\circ} \mathrm{C}$ overnight. Subsequently, apical buds were excised and prepared for light microscopy analysis.

BUD SAMPLE PREPARATION AND HISTOLOGY. Apical buds from fresh harvested uprights were excised and prepared for sectioning. Sample preparation was performed as described by Bolivar-Medina et al. (2019). Briefly, apical buds were fixed in a solution of glutaraldehyde (Sigma-Aldrich, St. Louis, MO) overnight at $4{ }^{\circ} \mathrm{C}$, rinsed in phosphate buffer, and dehydrated in a graded ethanol series. Subsequently, samples were slowly embedded by replacing ethanol with medium grade LR white resin (Ted Pella, Redding, CA). Once samples in resin were polymerized at $60{ }^{\circ} \mathrm{C}$ for $36 \mathrm{~h}$, they were mounted on stubs and cross-sectioned on an ultramicrotome (MT2; Sorvall, Norwalk, CT). Thin sections $(2 \mu \mathrm{m})$ were collected, mounted on Probe-On-Plus slides (Fisherbrand, Hampton, NH), and stained with $0.05 \%(\mathrm{w} / \mathrm{v})$ Toluidine Blue O (Sigma-Aldrich); coverslips were annealed with Cytoseal 60 (Richard Allan Scientific Co., San Diego, CA). All observations were performed on a bright field microscope (BX50; Olympus Optical Co., Tokyo, Japan) equipped with a digital camera (EOS Rebel T6i; Canon, Tokyo, Japan), and $10 \times / 0.30$, $20 \times / 0.50$, and $40 \times / 0.75$ objectives (magnification/numerical aperture) were used (Plan Apo; Olympus Optical Co.). 
For each set of buds collected from T0 to T4, at least five independent buds per category (VL, VM, VS, FM, FS) were cross-sectioned. The total number of floral meristems and their developmental stage were recorded from the equatorial crosssections. The number of floral meristems of a single bud can range from four to nine, and their stage of development varies. Therefore, for each evaluation, the floral meristem developmental stage was determined by the most advanced meristem identified. The average number of floral meristems and developmental stage per bud were summarized. The number of floral meristems and the floral meristem developmental stages were analyzed by a two-way analysis of variance using type III sums of squares.

Field Phenotypical evaluation. The sixth set of tagged uprights, which were left in the field until T5 (year 2, early fall), was evaluated to determine the reproductive status of the new uprights (vegetative or fruiting), total number of flowers that reached anthesis, and number of harvestable fruit per upright. The proportion of uprights with vegetative or fruiting reproductive status and the average number of flowers and fruit per upright were calculated. Because not all buds in the categories studied resulted in fruiting uprights, and because some categories had a sample size of five or fewer, the data were analyzed using the Fisher exact test for independence. $P$ values were calculated using Monte Carlo simulations. All statistical analyses were performed in the $\mathrm{R}$ environment ( $\mathrm{R}$ Core Team 2014).

UPRIGHT DEMOGRAPHICS. At T0 (year 1, preharvest), biomass samples were collected from three $929-\mathrm{cm}^{2}$ locations within the bed to determine the total upright density and proportions of VL, VM, VS, FM, and FS buds. Samples were collected from the same bed where uprights were tagged for the study and stored at $4{ }^{\circ} \mathrm{C}$ until analysis.

\section{Results}

Number of floral Meristems Per bud. Bud category and collection time are associated with the number of floral meristems per bud (Table 1). Except for the small buds on fruiting uprights (FS), there were similar numbers of floral meristems across bud sizes and uprights types (VL, VM, VS, and FM) when they reached T4 (year 2, late spring). However, there were differences in the numbers of floral meristems from period T0 to period T3 (Fig. 2). During these collection times, large buds from vegetative uprights (VL) contained the highest number of floral meristems, with medium buds from vegetative and fruiting uprights (VM and FM, respectively) containing the next highest number. In small buds from vegetative uprights (VS), floral meristems were visible for the first time by $\mathrm{T} 1$; in small buds of fruiting uprights (FS), they were visible for the first time by T2. The number of visible floral meristems in VS and FS continued to increase during the evaluation period, although FS had the lowest number compared with the other upright type/bud combinations (Fig. 2).

Developmental Stages of a Floral meristem. Reproductive or "mixed" buds differentiate floral meristems acropetally, resulting in floral meristems in a single bud that can be at different developmental stages. Developmental stages were determined based on the most advanced concentrically differentiated organ primordia. Five distinguishable developmental stages were identified (Fig. 3). When a floral initial emerges as a globular entity from the axil of a bud scale, two lateral bract

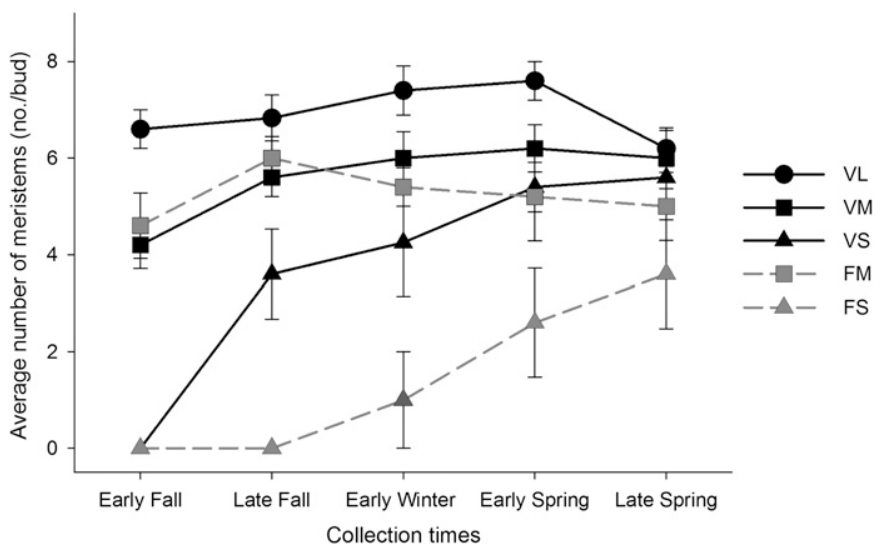

Fig. 2. Average number of floral meristems per bud from T0 (year 1, early fall) to T4 (year 2, late spring) for cranberry. Black lines correspond to buds on vegetative uprights $(\mathrm{V})$, and gray lines correspond to fruiting uprights $(\mathrm{F})$. Circles ( ) represent large buds $(\mathrm{L} ;>1.0 \mathrm{~mm})$, squares $(\boldsymbol{\square})$ represent medium buds (M; 0.6-1.0 $\mathrm{mm}$ ), and triangles $(\boldsymbol{\Delta})$ represent small buds $(\mathrm{S} ; \leq 0.5 \mathrm{~mm})$. Error bars represent the SE $(n=5)$.

primordia differentiate, with each on one side of the floral meristem and perpendicular to the embryonic shoot (stage 1) (Fig. 3A and B). Subsequent floral organ primordia are patterned in a whorled phyllotaxis. First, four sepal primordia differentiate: two laterals, one abaxial, and one adaxial (stage 2) (Fig. 3C). Next, four petal primordia form, appearing as arced triangle structures (stage 3 ) in an alternate pattern with the sepals and a square appearance on the floral meristem (Fig. 3D). Petal primordia elongate to cover the remaining floral meristem. Subsequently, eight anther primordia (the androecium) differentiate around the perimeter of the remaining central ground floral meristem (stage 4) (Fig. 3E), which finally gives rise to the gynoecium primordium (stage 5) (Fig. $3 \mathrm{~F})$.

Floral developmental STAGe Per bud during dormancy. Differences in floral developmental stages across upright type and bud size combinations were observed during the study period (Table 2). VL buds were already at developmental stage 4 at T0 and reached stage 5 by T1 (Fig. 4). VM buds were at stage 3 at T0 and reached stage 5 by T4. FM buds were between stages 2 and 3 at T0; by T4, they were intermediate between stages 3 and 4 . VS buds had no visible floral initials at T0, but by the following $\mathrm{T} 1$, they were between stages 2 and 3 ; subsequently, they were intermediate between stages 3 and 4 by T4. Finally, FS buds had no visible floral initials until T3, when buds were between stages 1 and 2; at T4, they had only reached developmental stage 2 (Fig. 4).

Phenotypical evaluation of UPRights Growing IN THE FIELD. An evaluation of the set of uprights collected at T5 showed that $100 \%$ of VL and VM buds and $85 \%$ of VS buds tagged at T0 produced new fruiting uprights during year 2 (Fig. 5 ), whereas FM and FS buds produced $85 \%$ and $38 \%$ of new fruiting uprights during year 2, respectively. Although all fruiting uprights produced flowers, not all set harvestable fruit (Fig. 6). Fisher's exact test indicated that the bud category is significantly associated with the number of flowers and berries ( $P=0.028$ and 0.008 , respectively). Uprights that grew from VL, VM, and VS buds produced more berries per upright than those from FM and FS buds. Particularly, uprights produced during year 2 from VL buds developed the highest average 

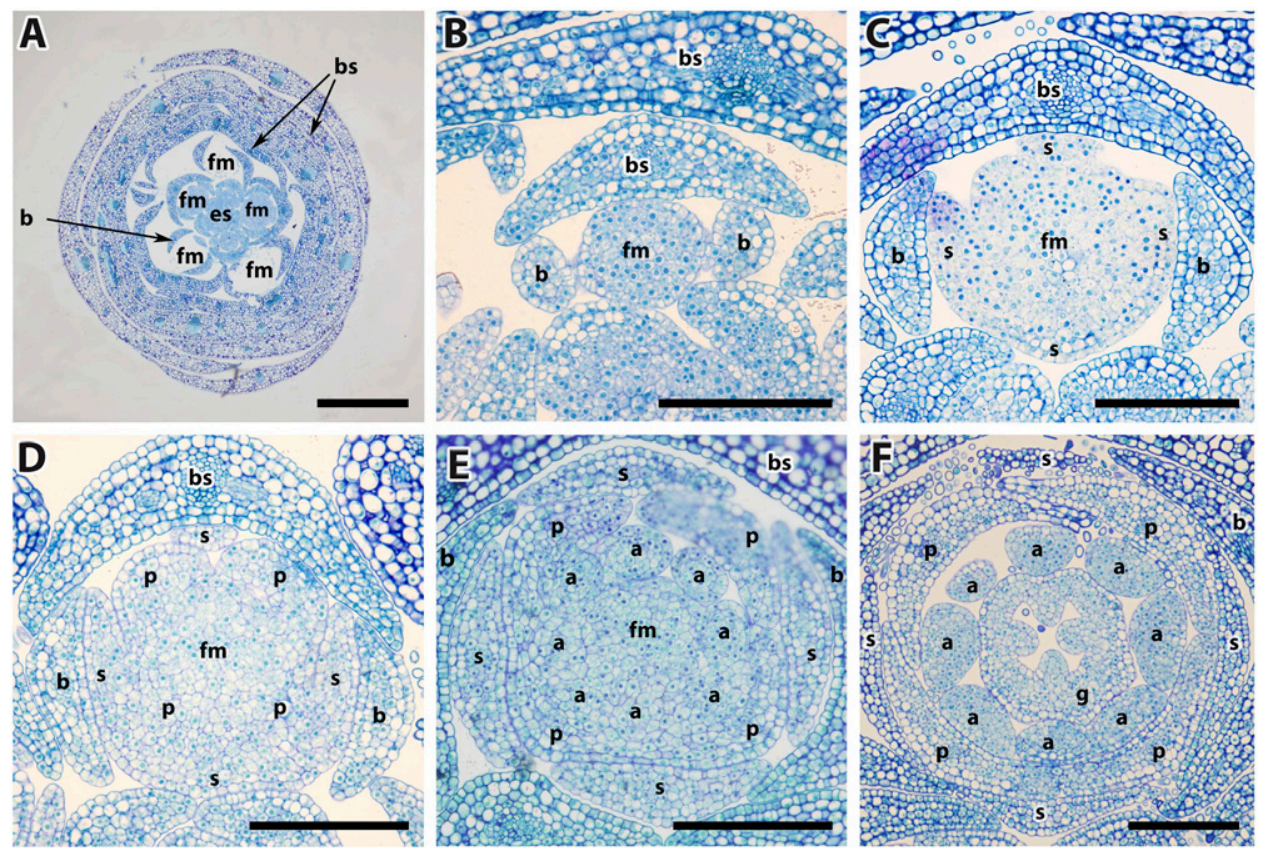

Fig. 3. Developmental stages of the floral meristems of the cranberry terminal bud. Examples depicted here are cross-sections from reproductive buds sampled at T0 (year 1, early fall). (A) Overview of the entire bud. Bud scales (bs) protect the bracts (b), floral meristems (fm), and the embryonic shoot (es). (B-F) Cross-sections of floral meristems at different stages of development. Floral meristems differentiate concentrically. (B) Stage 1: Floral meristem seen as a globular structure protected by one internal bud scale (bs) and two lateral bracts (b). (C) Stage 2: Four sepal primordia (s) differentiate on each side of the meristem. (D) Stage 3: Four petal primordia $(\mathrm{p})$ differentiate, giving a square appearance to the floral meristem. (E) Stage 4: Eight anther primordia and the androecium (a) surround the center of the meristem. (F) Stage 5: All primordia have differentiated, including the gynoecium $(\mathrm{g})$. Scale bars: $(\mathbf{A})=500 \mu \mathrm{m} ;(\mathbf{B}-\mathbf{F})=100 \mu \mathrm{m}$.

Table 2. Analysis of variance (type III) for floral meristem developmental stages per bud of cranberry during the dormant period from T0 (year 1, early fall) to T4 (year 2, spring 2016).

\begin{tabular}{lcrrl}
\hline Source & Sum of squares & df & $F$ & $P$ \\
\hline Intercept & 28.800 & 1 & 25.7483 & $1.823 \mathrm{e}-06^{* * *}$ \\
Bud category & 67.840 & 4 & 15.1629 & $1.056 \mathrm{e}-09^{* * *}$ \\
Collection time & 9.483 & 4 & 2.1196 & 0.08398 \\
Bud category: Collection time & 31.866 & 16 & 1.7806 & $0.04439^{*}$ \\
Residuals & 110.733 & 99 & & \\
\hline
\end{tabular}

*,**,***Significant at $P \leq 0.05,0.01$, or 0.001 , respectively

number of flowers (five) and berries (four) per upright of all categories evaluated. In contrast, uprights produced during year 2 from FM and FS buds produced the lowest average number of flowers (two to three) and berries per upright (one to two) (Fig. 6).

UPRIGHT DEMOGRAPHICS. Analysis of upright demographics from the $929-\mathrm{cm}^{2}$ area biomass samples collected at T0 showed that the proportion of vegetative uprights per 929 $\mathrm{cm}^{2}$ was two-times that of fruiting uprights (Table 3 ). The three bud size categories (large, medium, and small) were found only for vegetative uprights (VL, VM, and VS, respectively), and only medium and small buds were found for fruiting uprights (FM and FS). The most predominant bud size of the vegetative uprights was medium (VM; 45\%) compared with the proportion of vegetative uprights with large buds (VL; 28.3\%) and small buds (VS; 26.6\%). Within fruiting uprights, FS buds were more frequent $(86.7 \%)$ than FM buds (13.3\%).

\section{Discussion}

The present study documented the development of floral meristems in apical buds of vegetative and fruiting uprights in a new highyielding cranberry cultivar from fall to spring and considered their contributions to fruit production. Although several studies have focused on determining the presence and timing of the first appearance of floral initials in buds of vegetative and fruiting uprights of cranberry (Bolivar-Medina et al., 2019; DeVetter et al., 2013a), studies have not tracked development during the dormant period, and there has been no information regarding the differential contribution to fruit production.

Floral meristem differentiation in cranberry buds occurs acropetally (Bolivar-Medina et al., 2019), as does the pattern of anthesis (Brown and McNeil, 2006). In our study, basal floral meristems had the most advanced development across all upright type and bud size combinations, and they were the greatest contributors to fruit production during the next growing season. It is possible that less developed floral meristems in upper positions serve as pollen sources for fully developed flowers in lower positions, or they might serve as a backup in case lower-position flowers fail to set fruit (Brown and McNeil, 2006). We established five stages of floral meristem development (Fig. 3) based on the differentiation of organ primordia. These primordia developed in a whorled pattern characteristic of most eudicots, including highbush blueberry (Vaccinium corymbosum) (Kovaleski et al., 2015) and Rhododendron (Mirgorodskaya et al., 2015), and other perennial fruits, such as blackberry (Rubus subgenus Rubus) (Takeda et al., 2002), and sweet cherry (Prunus avium) (Fadón et al., 2015).

Although the external appearance of apical buds remained unchanged during the dormant period (fall through early spring), the histological analysis revealed differences in timing, developmental stages attained, and number of floral meristems across bud sizes and upright types (Figs. 2 and 4). Previous histological studies of cranberry apical buds reported that before harvest in the early fall (T0), floral initials formed and differentiated into floral meristems in large buds of vegetative and fruiting uprights (Bolivar-Medina et al., 2019; DeVetter et al., 2013a; Goff, 1901; Lacroix, 1926). Our observations not only support these previous findings but also show that floral meristems are differentiated in large, medium, and small buds 


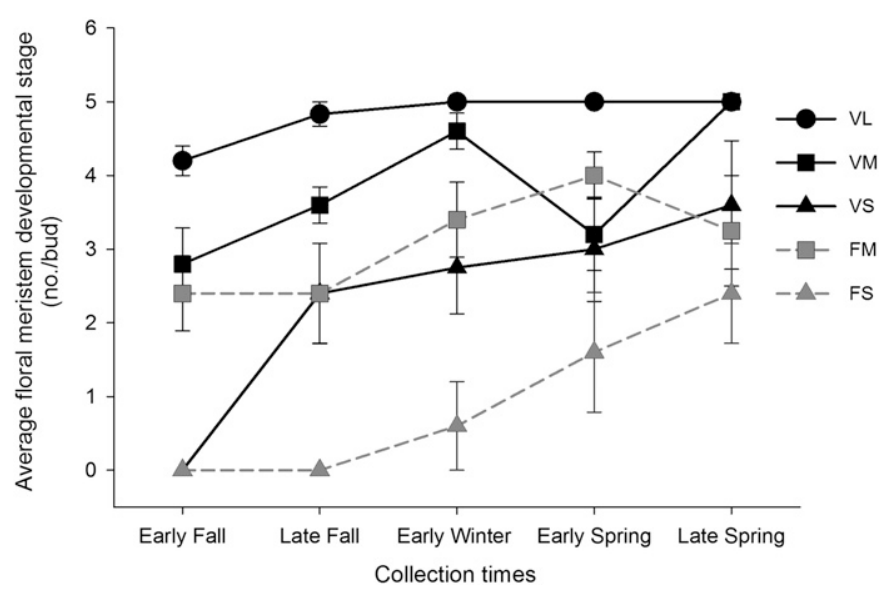

Fig. 4. Average developmental stage of floral meristems per bud from T0 (year 1 , early fall) to T4 (year 2, late spring) for cranberry. Black lines correspond to buds on vegetative uprights (V), and gray dashed lines correspond to fruiting uprights $(\mathrm{F})$. Circles $(\mathbf{O})$ represent large buds $(\mathrm{L} ;>1.0 \mathrm{~mm})$, squares $(\boldsymbol{\square})$ represent medium buds $(\mathrm{M} ; 0.6-1.0 \mathrm{~mm})$, and triangles $(\boldsymbol{\Delta})$ represent small buds $(S ; \leq 0.5 \mathrm{~mm})$. Error bars represent the $\mathrm{SE}(\mathrm{n}=5)$.

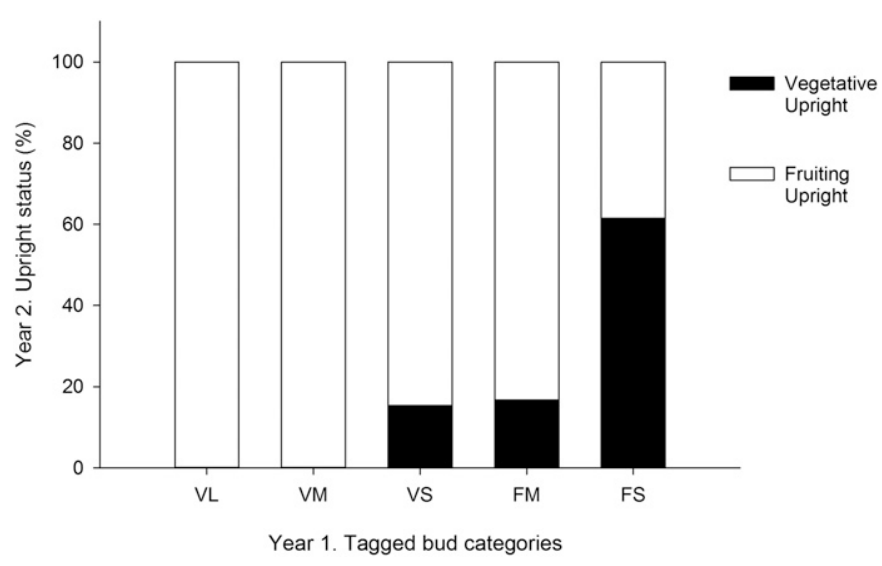

Fig. 5. Percentage of vegetative and fruiting cranberry uprights at T5 (year 2, early fall) that grew from buds tagged at T0 (year 1, early fall). T0 buds were categorized by the current upright status [vegetative $(\mathrm{V})$ or fruiting $(\mathrm{F})$ ] and bud width size: large $(\mathrm{L} ;>1.0 \mathrm{~mm})$, medium $(\mathrm{M} ; 0.6-1.0 \mathrm{~mm})$, and small $(\mathrm{S} ; \leq 0.5 \mathrm{~mm})$. Areas of bars correspond to the percentages of the resulting vegetative (black) and fruiting (white) uprights (VL, $\mathrm{n}=11 ; \mathrm{VM}, \mathrm{n}=10$; VS, $\mathrm{n}=13 ; \mathrm{FM}, \mathrm{n}=12 ; \mathrm{FS}, \mathrm{n}=13$ ).

of both fruiting and vegetative uprights from fall to spring (Fig. 2 ). The number of floral meristems is lower for small buds compared to large buds, and for the buds of fruiting compared to vegetative uprights (Fig. 2). The higher number of floral meristems observed in buds from vegetative compared to fruiting uprights is likely due to the unrestricted allocation of photosynthates produced by the vegetative upright. On the contrary, fruiting uprights must partition photosynthates between the stronger fruit sink and the buds (Roper and Klueh, 1994, 1996), thus limiting and/or delaying floral differentiation. In addition, the endogenous plant growth regulator production of developing fruit may affect the timing of floral meristem development, as reported for Citrus species (Goldschmidt et al., 1985; Koshita et al., 1999).

During late fall (T1), VL and VM buds had largely completed differentiation of the basal flowers (Fig. 4). It is possible that the androecium and gynoecium of floral meristems of VL continued their internal differentiation during fall and winter, as in peach (Prunus persica) (Reinoso et al., 2002). In addition, these uprights may accumulate starch to support the flowering process in spring, as in sweet cherry and apricot (Prunus armeniaca) (Fadón et al., 2015; Fadón et al., 2017; Julian et al., 2011). Alternatively, during late fall, the FS, FM, and VS buds were only at the initial stages of floral meristem development, with most of their floral primordia differentiation process occurring through winter (Fig. 4). The delay in appearance of the first floral initials could be explained by differences in the carbohydrate content of the upright types. A previous study of the cranberry upright carbohydrate content reported an increase in upright carbohydrate levels from fruit set until fall, with higher rates of accumulation for vegetative compared to fruiting uprights (DeVetter et al., 2016; Hagidimitriou and Roper, 1994). This difference in accumulation rates would explain the earlier appearance of floral meristems in VL and VM compared to FM and FS. Differences in the timing of differentiation between large and small budded vegetative uprights (Figs. 2 and 4) are probably due to lower photosynthate production by less vigorous uprights with both fewer and smaller leaves, resulting in less support for the differentiation of floral meristems. It is interesting to note how late VS and FS buds start developing floral meristems (Fig. 2). It is possible that, similar to the tuberous species Corydalis bracteata, chilling temperatures in late fall and winter activate the apoplastic movement of carbohydrates from the leaves and stems to the apical buds, acting as signal molecules for the differentiation and development of floral meristems (Khodorova et al., 2010). In addition, with the warming conditions in early spring, photosynthates produced by the leaves of the previous year (Hagidimitriou and Roper, 1994) may be transported to the VS and FS buds, supporting the differentiation of the floral meristems before budbreak. Additional studies of the carbohydrate content of the apical buds of cranberry through the dormant period would contribute to the understanding of the role of carbohydrate translocation during fall and winter floral meristem differentiation.

Our results showed that flower production and fruit production are highly dependent on the upright type and bud set of the previous year. All VL and VM buds and most VS buds grew as fruiting uprights (Fig. 5). VL buds had the resources to differentiate the highest number of floral meristems before winter, which resulted in the highest number of flowers and fruit produced per upright across all upright type and bud size combinations (Fig. 6). VM and VS buds had comparable fruit production per upright (Fig. 6), despite the late floral differentiation of the latter (Fig. 4). Interestingly, FM produced fewer fruit per upright than VS, despite having produced similar numbers of floral meristems and developing earlier in the fall. In fact, buds from fruiting uprights had a minor contribution to fruit production compared with those from vegetative uprights, and although they do produce flowers and are considered nonalternate bearing (Bolivar-Medina et al., 2019; Clark and Finn, 2010; DeVetter et al., 2013b; McCown and Zeldin, 2003), the flowers produced by FM and FS buds have a low fruit set. Despite the differences in fruit production per upright, the size and shape of the fruit were unaffected by the upright type or bud size categories studied. It is possible that there is a trade-off between the carbohydrate allocation and the number of berries 


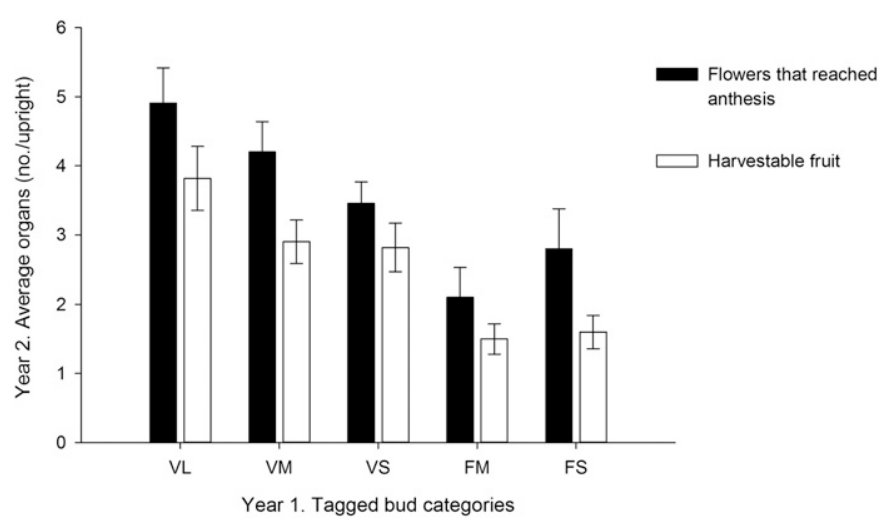

Fig. 6. Average number of flowers and berries on cranberry fruiting uprights at $\mathrm{T} 5$ (year 2, early fall) produced from buds tagged at $\mathrm{T} 0$ (year 1, early fall). T0 buds were categorized by the current upright status [vegetative $(\mathrm{V})$ or fruiting (F)] and bud width size: large (L;>1.0 mm), medium (M; 0.6-1.0 mm), and small $(\mathrm{S} ; \leq 0.5 \mathrm{~mm})$. White bars correspond to the number of flowers per upright that reached anthesis (identified by pedicels ranging from those with a flower that did not result in a developed fruit to those with attached harvestable fruit). Solid bars correspond to attached harvestable fruit. Error bars represent the SE $(V L, n=11 ; \mathrm{VM}, \mathrm{n}=10 ; \mathrm{VS}, \mathrm{n}=11 ; \mathrm{FM}, \mathrm{n}=10 ; \mathrm{FS}$, $\mathrm{n}=5$ ).

Table 3. Percentage of vegetative and fruiting uprights and bud size categories per $929-\mathrm{cm}^{2}$ area present at T0 (year 1 , early fall) in a commercial bed of 'HyRed' cranberry located near Necedah, WI.

\begin{tabular}{lc}
\hline Upright type & Uprights per $929 \mathrm{~cm}^{2}(\%)$ \\
\hline Vegetative uprights $(\mathrm{V})$ & 64.12 \\
VL & 28.32 \\
VM & 45.07 \\
VS & 26.61 \\
Fruiting uprights (F) & 35.88 \\
FL & 0.00 \\
FM & 13.30 \\
FS & 86.70
\end{tabular}

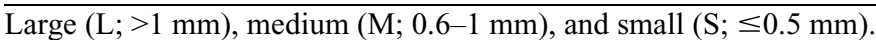

that an upright can successfully develop without affecting its shape and size.

Our work evaluated the development of floral meristems from fall to spring in buds of different sizes and upright types and their contribution to fruit production. Apical buds of cranberry actively differentiate floral meristems during fall and winter, and there are differences in the timing of development based on bud size. We also found that buds from vegetative uprights are critical for the fruit production of the next growing season because those buds become the fruiting uprights with the highest number of fruit. Even though new cranberry cultivars, like HyRed, are considered nonalternate bearing, their consistent yields are determined more by the production of new vegetative uprights rather than the return blooming of pre-existing fruiting uprights. This study highlighted the need to re-evaluate yield prediction models of cranberry, particularly the components of upright type and bud size.

\section{Literature Cited}

Aggelopoulou, A.D., D. Bochtis, S. Fountas, K.C. Swain, T.A. Gemtos, and G.D. Nanos. 2011. Yield prediction in apple orchards based on image processing. Precis. Agr. 12:448-456.
Alvarez-Buylla, E.R., M. Benítez, A. Corvera-Poiré, A. Chaos, S. de Folter, A. Gamboa de Buen, A. Garay-Arroyo, B. Garcia-Ponce, F. Jaimes-Miranda, R. Perez-Ruiz, A. Piñeyro-Nelson, and Y.E. Sanchez-Corrales. 2010. Flower development. Arabidopsis Book 8:e1027, doi: 10.1199/tab.0127.

Bolivar-Medina, J.L., J.E. Zalapa, A. Atucha, and S.E. Patterson. 2019. Relationship between alternate bearing and apical bud development in cranberry (Vaccinium macrocarpon). Botany 97:101-111.

Brown, A.O. and J.N. McNeil. 2006. Fruit production in cranberry (Ericaceae: Vaccinium macrocarpon): A bet-hedging strategy to optimize reproductive effort. Amer. J. Bot. 93:910-916.

Clark, J.R. and C.E. Finn. 2010. Register of new fruit and nut cultivars list 45. HortScience 45:716-756.

DeVetter, L., E. Beaver, J. Colquhoun, J.E. Zalapa, and R. Harbut. 2016. Comparison of nonstructural carbohydrates across cranberry cultivars. Eur. J. Hort. Sci. 81:321-326.

DeVetter, L., J. Colquhoun, J.E. Zalapa, and R. Harbut. 2015. Yield estimation in commercial cranberry systems using physiological, environmental, and genetic variables. Scientia Hort. 190:83-93.

DeVetter, L., R. Harbut, and J. Colquhoun. 2013a. Bud development, return bloom, and external bud appearance differ among cranberry cultivars. J. Amer. Soc. Hort. Sci. 138:338-343.

DeVetter, L., R. Harbut, and J. Colquhoun. 2013b. Understanding yield-contributing factors of cranberry (Vaccinium macrocarpon Ait.). J. Am. Pomol. Soc. 67:147-156.

Fadón, E., M. Herrero, and J. Rodrigo. 2015. Flower development in sweet cherry framed in the BBCH scale. Scientia Hort. 192:141-147.

Fadón, E., M. Herrero, and J. Rodrigo. 2017. Flower bud development and chilling requirements in 'Bing' sweet cherry. Acta Hort. 1161:361-366.

Goff, E.S. 1901. Investigations of flower buds. Annu. Rpt. Agr. Expt. Stn. Univ. Wisconsin 18:304-319.

Goldschmidt, E.E., N. Aschkenazi, Y. Herzano, A.A. Schaffer, and S.P. Monselise. 1985. A role for carbohydrate levels in the control of flowering in citrus. Scientia Hort. 26:159-166.

Hagidimitriou, M. and T. Roper. 1994. Seasonal changes in nonstructural carbohydrates in cranberry. J. Amer. Soc. Hort. Sci. 119:1029-1033.

Julian, C., J. Rodrigo, and M. Herrero. 2011. Stamen development and winter dormancy in apricot (Prunus armeniaca). Ann. Bot. 108:617625.

Khodorova, N.V., E.A. Miroslavov, A.L. Shavarda, and J. Laberche. 2010. Bud development in corydalis (Corydalis bracteata) requires low temperature: A study of developmental and carbohydrate changes. Ann. Bot. 105:891-903.

Koshita, Y., T. Takahara, T. Ogata, and A. Goto. 1999. Involvement of endogenous plant hormones (IAA, ABA, GAs) in leaves and flower bud formation of satsuma mandarin (Citrus unshiu Marc.). Scientia Hort. 79:185-194.

Kovaleski, A.P., J.G. Williamson, J.W. Olmstead, and R.L. Darnell. 2015. Inflorescence bud initiation, development, and bloom in two southern highbush blueberry cultivars. J. Amer. Soc. Hort. Sci. 140:38-44.

Lacroix, D.S. 1926. Cranberry flower-bud investigations. J. Agr. Res. 33:355-363.

Liu, C., Z. Thong, and H. Yu. 2009. Coming into bloom: The specification of floral meristems. Development 136:3379-3391.

McCown, B.H. and E.L. Zeldin. 2003. 'HyRed', an early, high fruit color cranberry hybrid. HortScience 38:304-305.

Mirgorodskaya, O.E., N.K. Koteyeva, A.V. Volchanskaya, and E.A. Miroslavov. 2015. Pollen development in Rhododendron in relation to winter dormancy and bloom time. Protoplasma 252:1313-1323.

Polito, V.S., K. Pinney, R. Heerema, and S.A. Weinbaum. 2002. Flower differentiation and spur leaf area in almond. J. Hort. Sci. Biotechnol. 77:474-478.

R Core Team. 2014. R: A language and environment for statistical computing. R Foundation for Statistical Computing, Vienna, Austria. 
Reinoso, H., V. Luna, R.P. Pharis, and R. Bottini. 2002. Dormancy in peach (Prunus persica) flower buds. V. Anatomy of bud development in relation to phenological stage. Can. J. Bot. 80: 656-663.

Roberts, R.H. and B.E. Struckmeyer. 1943. Blossom induction of the cranberry. Plant Physiol. 18:534-536.

Roper, T. and J.S. Klueh. 1994. Removing new growth reduces fruiting in cranberry. HortScience 29:199-201.

Roper, T. and J.S. Klueh. 1996. Movement patterns of carbon from source to sink in cranberry. J. Amer. Soc. Hort. Sci. 121:846-847.
Roper, T. and N. Vorsa. 1997. Cranberry: Botany and horticulture. Hort. Rev. 21:215-249.

Takeda, F., B.C. Strik, D. Peacock, and J.R. Clark. 2002. Cultivar differences and the effect of winter temperature on flower bud development in blackberry. J. Amer. Soc. Hort. Sci. 127:495-501.

Trehane, J. 2004. Blueberries, cranberries and other vacciniums. Timber Press, Portland, OR.

Vorsa, N. and J. Johnson-Cicalese. 2012. American cranberry, p. 191223. In: M.L. Badenes and D.H. Byrne (eds.). Fruit breeding, handbook of plant breeding 8 . Springer, Boston, MA. 